- New drivers: A shift from innovation to eco-innovation requires new drivers with an explicit focus on eco-efficiency. Drivers or incentives to bring about these changes include the use of communication tools (green marketing or advertising, education, awareness raising campaigns), legislation, pricing and other governmental policies (such as on environment, labour, transport). Individual consumers should be empowered to become concerned citizens. Research on consumer behaviour was considered important, as well as research on pricing as an instrument for social change, and on specific needs and perspectives for developing countries, more in particular on their abilities to achieve eco-innovation, supported by fair technology transfer. A long-list of detailed issues for further research was approved.

\section{《 》}

\section{„Die Asse säuft ab - Gorleben was nun?"}

Bericht von der „Fachtagung zum Salzstock"

Dannenberg, 16.-17. April 2010

von Sophie Kuppler, ITAS

\section{Hintergrund}

Welche Argumente sprechen gegen eine Nutzung des Salzstocks Gorleben für die tiefengeologische Endlagerung hochradioaktiver Abfälle? Diese Frage prägte die von der Bürgerinitiative Umweltschutz Lüchow-Dannenberg organisierte „Fachtagung zum Salzstock“, für die sich ca. 200 Teilnehmer vom 16. und 17. April in Dannenberg im Wendland einfanden. Die am 2. März 1977 gegründete Bürgerinitiative ist ein Zusammenschluss von über 1.000 Atomkraftgegnern und hat einen sofortigen Ausstieg aus der Kernenergie zum Ziel. ${ }^{1}$ Sie engagiert sich maßgeblich gegen die Ernennung des Salzstocks Gorleben als Endlager für hochradioaktive Abfälle.
Anlass, zehn Jahre nach der letzten von der Bürgerinitiative finanzierten Fachtagung Experten aus unterschiedlichen wissenschaftlichen Disziplinen und Organisationen zusammenzubringen, waren die öffentlichen Debatten um die mögliche Einflussnahme von Politikern auf wissenschaftliche Ergebnisse im Auswahlprozess für den Endlagerstandort und die Aufhebung des Gorleben-Moratoriums durch die Bundesregierung. ${ }^{2}$ Diese und andere Ereignisse der letzten Jahre sollten von Experten analysiert und bewertet werden. Ziel der Tagung mit dem Titel „Die Asse säuft ab - Gorleben was nun?" war, die wissenschaftliche Basis der Argumentation gegen den Salzstock Gorleben als Endlager zu erweitern. Neben geologischen Aspekten der Standorteignung spielte die Frage der Angemessenheit des Standortauswahlverfahrens eine zentrale Rolle, die sowohl aus historischer als auch ethischer Sicht diskutiert wurde. Die vortragenden Experten waren entweder universitär oder privatwirtschaftlich tätig, oder einer bürgerschaftlichen Organisation zuzuordnen.

\section{Historische Erkenntnisse über die Aus- wahlverfahren für Asse II und Gorleben}

Der Historiker Detlev Möller belegte anhand von Zitaten aus Originalakten die frühe Festlegung von Asse II als Endlager bis zum Jahr 2000, obwohl die hohe Gefahr eines Wassereinbruchs bereits bekannt gewesen sei. Selbst nach dem Entdecken von Deformationen im Gestein 1977 sei zwar die Handlungsnotwendigkeit erkannt, aber nichts getan worden. Eine Übertragbarkeit der Erfahrungen mit Asse II auf den Standort Gorleben sah Möller nur indirekt dadurch, dass Asse II die einzige Probemöglichkeit für die Endlagerung in Salz gewesen sei, auf deren Basis die Genehmigung für Gorleben erteilt werden sollte.

Matthias Edler, Atomexperte bei Greenpeace, präsentierte die Ergebnisse einer Aktenschau zum Auswahlverfahren des Standorts Gorleben. Gegenstand dieser Aktenschau waren die Studie der Kernbrennstoff-WiederaufbereitungsGesellschaft (KEWA), die im Auftrag des Bundesministeriums für Forschung und Technologie von 1974 bis 1976 durchgeführt wurde, die Studie des Interministeriellen Arbeitskreises der niedersächsischen Landesregierung (IMAK; 08/1976- 
02/1977) und des TÜV (06/1976-12/1976). Diese Studien dienten der Auswahl eines Standorts für ein nukleares Entsorgungszentrum, weshalb neben geologischen Kriterien für ein Endlager weitere, auf die Industrieanlagen des geplanten Entsorgungszentrums bezogene Kriterien eine wichtige Rolle gespielt hätten. Die TÜV-Studie hätte sogar ganz auf geologische Kriterien verzichtet. Es sei nicht mehr nachzuvollziehen, von wem und unter welchen Umständen Gorleben als weiterer Standort handschriftlich zur TÜV-Studie hinzugefügt worden sei. Edler betonte weiterhin den Zeitdruck, unter dem die Entscheidung damals gefälltt worden sei, da die Lagerungskapazitäten der Energieversorgungsunternehmen knapp geworden und damit der Weiterbetrieb der Kernkraftwerke gefährdet gewesen wären. Die Dringlichkeit der Entscheidung sei in mehreren Briefen des damaligen Bundeskanzlers Helmut Schmidt an den niedersächsischen Ministerpräsidenten Ernst Albrecht betont worden.

\section{Geologische Argumente gegen die Eig- nung von Gorleben und Anforderungen an ein Auswahlverfahren}

Ulrich Schneider befasste sich in seinem Vortrag mit geologischen Fragen der Standortsicherheit, insbesondere mit der Gefahr eines unkontrollierten Wasserzuflusses in das geplante Endlager. Er wies auf die von der „Bundesanstalt für Geowissenschaften und Rohstoffe" (BGR) bestätigten Scheitelstörungen hin und die damit verbundene Gefahr eines Wassereintritts und Radionuklidaustritts. Es bestehe eine fortwährende Grundwasserneubildung, was bedeute, dass Oberflächenwasser in tiefere Schichten, bis hin zum Salzspiegel, eintrete und eine Bewegung quer über den Salzstock hinweg stattfinde. Ein weiteres Problem sei, dass für die Erkundung des Salzstocks der Hauptanhydrit mehrfach durchfahren werden müsse, was ein Sicherheitsrisiko darstelle. Schneider forderte, dass BfS und BGR sich widersprechende Daten zur Ausdehnung von Laugenreservoiren in ihren Publikationen klären.

Auch Dieter Ortlam ging auf den Wasserfluss über den Salzstock hinweg ein, der schon seit Ende der 1960er Jahre bekannt sei. Das Vorhandensein von Salzwasser an der Oberfläche in der Nähe von Gorleben erklärte er mit Hilfe des DGH-Effektes (Tauchgleichgewicht). Dieser bewirke, dass durch die „Gorlebener Rinne“ Salzwasser aufsteige. Damit sei der Salzstock nicht für die Endlagerung geeignet.

Der Kulturhistoriker Ulrich Reiff berichtete über historische Kalibohrungen im Salzstock Gorleben Anfang des 20. Jahrhunderts. Die Gewerkschaft Nordenhall habe dort fünf Bohrungen vorgenommen und dabei teilweise den Salzstock verritzt. Eine Ausbeutung der Kalisalze sei nicht mehr zustande gekommen, allerdings seien bei der Verfüllung der Bohrlöcher Fehler unterlaufen, so dass z. B. teilweise noch Rohre in den Bohrlöchern steckten. Weiterhin in Betracht zu ziehen sei die Tatsache, dass im Kalibergwerk Wendland Einbrüche von Gas und Laugen auch in großen Tiefen Probleme bereitet hätten und weitere Bohrungen der Kaliwerke Teutonia in Richtung Wendland aufgrund von Laugeneinbrüchen abgebrochen worden seien. Welche Bedeutung diese Funde für die Bewertung der Eignung des Salzstocks als Endlager hätten, müsse von Geologen beurteilt werden.

Eckhard Kruse, Umweltbeauftragter der Evangelischen Landeskirche Hannover, referierte über ethische Anforderungen an die Endlagerung radioaktiver Stoffe. Im Fall der Endlagerung sei aufgrund des bereits vorhandenen Mülls nicht die Frage relevant, ob etwas ethisch (un-) bedenklich sei, sondern man müsse sich der Frage des Umgangs mit dem Falschen stellen. Die Evangelische Landeskirche Hannover fordere deshalb Beteiligung, Transparenz, standortunabhängige Kriterien und den Vergleich mehrerer Standorte und Lagermedien.

Jürgen Kreusch befasste sich in seinem Vortrag mit der Begründung der Bundesregierung für die Weitererkundung des Standorts Gorleben, wie sie der Pressemitteilung 037/10 des Bundesministeriums für Umwelt, Naturschutz und Reaktorsicherheit vom 17. März 2010 zu entnehmen ist. Er kam zu dem Schluss, dass die angebrachten Gründe für die Weitererkundung Gorlebens weder aus naturwissenschaftlich-technischer, noch gesellschaftlicher Sicht tragfähig seien und deshalb keine Grundlage für eine Aufhebung des Moratoriums bestünde. So sei das jetzige Verfahren nicht geeignet, um bestehende Defizite im 
Auswahlverfahren zu beheben: Der internationale Peer-review-Prozess diene beispielsweise nur der Bestätigung der Eignung, nicht der Kritik. Weiterhin sei der Terminus der „Eignungshöffigkeit" interpretationsleitend bei der Datenauswertung, so dass von ergebnisoffener Erkundung nicht gesprochen werden könne; dies ginge weiterhin nur, wenn Standortkriterien schon im Vorfeld festgelegt würden. ${ }^{3}$

\section{Fazit}

Die Tagungsbeiträge gaben einen guten Überblick über die in der wissenschaftlichen und politischen Debatte gegen Gorleben als Standort für ein Endlager vorgebrachten Argumente. Die vielfältigen Hintergründe der Vortragenden führten dabei zu einer Argumentation auf sehr unterschiedlichen Ebenen. In den wissenschaftlich orientierten bis hin zu rein politischen und journalistischen Vorträgen war eine Vielfalt von Argumentationen zu beobachten. Diese Vielfalt erschwerte einerseits Diskussionen, zeigte aber andererseits sehr deutlich die hohe Komplexität und weite gesellschaftliche Fassung des Problems der Endlagersuche, die durch eine rein natur- und technikwissenschaftliche Debatte nicht gelöst werden kann.

Diese Komplexität äußerte sich auch in zwei während der Tagung von Referenten und Teilnehmern mehrfach geäußerten Kritikpunkten: Erstens wurde beklagt, dass die Arbeit von „GegenExperten" sowohl in der Beurteilung der geologischen Eignung des Salzstocks Gorleben als auch in der Rekonstruktion des historischen Auswahlverfahrens durch den eingeschränkten Datenzugang behindert werde. Dies erschwere eine sachgerechte Beurteilung der Fakten und damit eine Diskussion auf Augenhöhe mit den von der Bundesregierung beauftragten Experten. Diese Diskussionen seien aber erforderlich, da das Vertrauen in Staatshandeln und Behörden aufgrund der geschilderten Erkenntnisse verloren gegangen sei und Intransparenz deshalb nicht geduldet werden könne. Zweitens wurde negativ vermerkt, dass Befürworter der Erkundung in Gorleben bzw. der momentan geplanten Vorgehensweise, nicht der Einladung zur Tagung nachkamen, d. h. sich der Diskussion nicht stellten (mit Ausnahme eines
Vertreters des Bundesamtes für Strahlenschutz). Dies zeige fehlende Offenheit für Argumente.

Dieser einseitigen Besetzung der Tagung entsprechend wurden die Beiträge wenig kontrovers diskutiert. Eine differenzierte geowissenschaftliche Auseinandersetzung wäre allerdings im Rahmen der Tagung ohnehin schwer möglich gewesen, da sie nicht der Zielsetzung und Zielgruppe entsprach. Positiv ist deshalb das in der Diskussion getroffene Vorhaben zu vermerken, eine separate Diskussionsveranstaltung zwischen BfS, BGR und bei der Tagung anwesenden Geologen zu organisieren. Sollte diese Veranstaltung zustande kommen, wäre dies ein wichtiger Schritt in der Diskussion um Interpretationsrahmen und Gewichtungen von Forschungsergebnissen.

\section{Anmerkungen}

1) http://www.bi-luechow-dannenberg.de/ueber-uns/ geschichte und http://www.bi-luechow-dannenberg.de/ueber-uns/unsere-ziele

2) Im Jahr 2000 wurde von der damaligen Bundesregierung die Erkundung des Salzstocks Gorleben in Vereinbarung mit den Energieversorgungsunternehmen für längstens zehn Jahre gestoppt, um in diesem Zeitraum grundsätzliche Fragen zur Endlagerung und zum Salzstock zu klären. Die zehn Jahre laufen im Sommer 2010 aus (s. auch Pressemitteilung Nr. 037/10 des Bundesministeriums für Umwelt, Naturschutz und Reaktorsicherheit).

3) $\mathrm{Zu}$ den Themen ,Atommüll in Bewegung - Die Endlagerung hochradioaktiver Abfälle gibt es noch nirgendwo" (Herman Damfeld) und „Die Asse säuft ab - ein Lehrstück für Gorleben“ (Rolf Bertram) gab es ebenso Vorträge, auf die hier nicht eingegangen wird. 